\title{
HYBRID BIOMIMETIC DIRECTIONAL MICROPHONE FOR THE FULL SPACE SOUND SOURCE LOCALIZATION
}

\author{
C. C. Chen ${ }^{*}, Y$. C. Chen, Keng-Yu Lin, and Y. T. Cheng \\ Microsystems Integration Laboratory, Department of Electronics Engineering \& Institute of Electronics, \\ National Chiao Tung University, Hsinchu, Taiwan, ROC.
}

\begin{abstract}
The paper presents a hybrid biomimetic directional microphone with a central floating pivot support by hybridizing the supersensitive ears of the parasitoid fly with the flexibility of the clover stalk. By introducing the mIIDpA, the mIPD, and the sensing region, the presented design with the state-of-the-art characteristics of mIIDpA $2.7 \mathrm{~dB} / \mathrm{mm}^{2}$ and $\mathrm{mIPD} 155^{\circ}$ has shown a highly potential application for the sound source localization with full space sensitivity. Excellence match between theoretical calculation and measurement results indicates the accuracy of the presented model and about 30\% net diaphragm displacement improvement.
\end{abstract}

\section{INTRODUCTION}

It has been a challenge using a miniature microphone to realize sound source localization due to diminutive interaural level difference and time difference [1]. Several developed biomimetic MEMS microphones still exhibited several deficiencies, such as the trade-off of structural sensitivity and rigidity and the increase of process complexity with optical readout integration $[1,2,4]$. Among them, the biomimetic microphone using a central pivot-supported (CP-S) design [3] has drawn lots of attention due to the characteristics of simple fabrication process, easy sensing circuit implementation, and good directional identification as well as signal sensing ability. However, the asymmetrical beam structure, shown in Fig. 1 (a), would lead to an undesired deformation that might cause device failure or the degradation of device sensitive and directional abilities. In order to overcome this dilemma, we present a new symmetrical beam structure. By hybridizing the supersensitive ears of the parasitoid fly with the flexibility of the clover stalk, as shown in Fig. 1 (b) and (c), the structurally coupled mechanism of the central floating support reveals two unique design merits: (1) fourfold rotation axial symmetry of the central beams effectively compensates the undesired deformation due to the gravity and the residual stresses for maintaining diaphragm rigidity, and (2) the central floating joint makes the sensing diaphragm more flexible for higher sound sensitivity and directivity.

For fair comparison, two microphones with the hybrid and $\mathrm{CP}-\mathrm{S}$ designs both having the same structure parameters listed in Table 1 are fabricated using a three-layer copper electroplating process [5] respectively to validate device performance. Measured resonant frequencies of the hybrid and CP-S designs are about 10 and $12 \mathrm{KHz}$, respectively, close to the CoventorWare simulation [6] shown in Fig. 2 (a). Better directivity and larger net displacement in the polar plot shown in Fig. 2 (b) reveal that the hybrid design has a superior ability in sound source localization, i.e. about $36 \%$ sensitivity and $34 \%$ directivity improvements in comparison with that of the CP-S design. Figure 3 shows measured acoustic responses of the hybrid microphone applied with $80 \mathrm{~dB}$ sound waves in frequency domain and it evidences that the hybrid design has well-performance at $200 \mathrm{~Hz}$ with significant phase difference. Figure 4 shows the diaphragm displacements of the two kinds of microphones driven by a $80 \mathrm{~dB}$ and $200 \mathrm{~Hz}$ sinusoidal sound wave located at $(r, \theta, \varphi)=\left(24 \mathrm{~cm}, 37^{\circ}, 0^{\circ}\right)$.

Meanwhile, by considering the motion equation of the microphone diaphragm, the corresponding displacement in the steady state of the ipsi- $(+)$ and contralateral $(-)$ membranes can

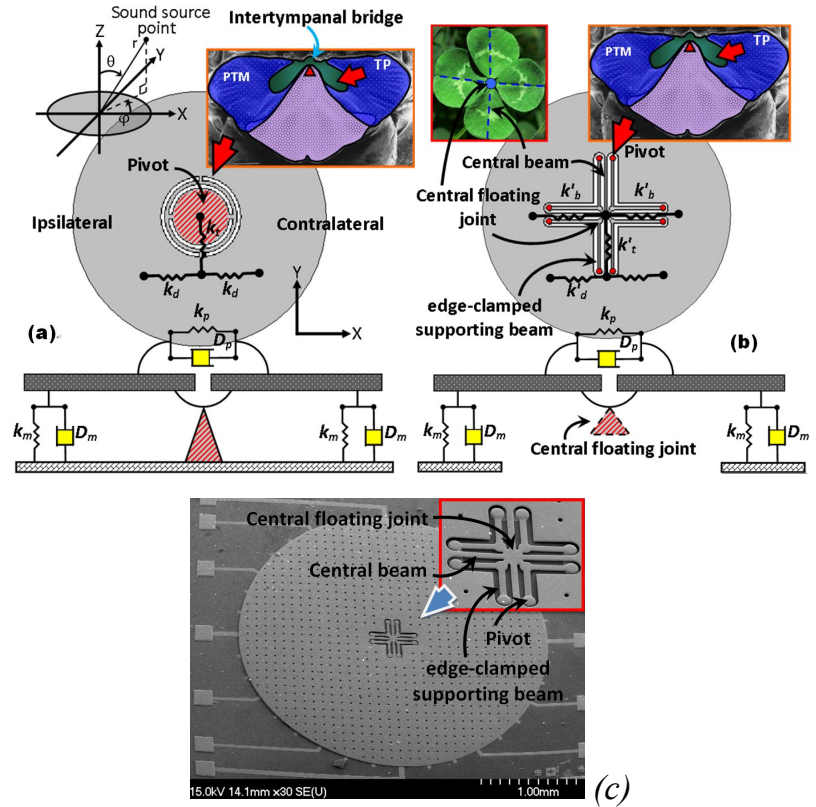

Figure 1: (a) Schemes of the conventional CP-S design with coordinate parameters $(r, \theta, \varphi)$. (b) Scheme of the hybrid biomimetic microphone with central floating gimbal design. (c) The SEM photograph of the hybrid microphone.

be expressed by the linear combination of the displacements of translational and rocking modes:

$$
\begin{aligned}
& Z_{ \pm}= \frac{P(t) \cdot \pi\left(a^{2}-c^{2}\right)}{m} \times \\
&\left\{\frac{\cos (\omega \tau / 2) \sin \left(\omega t+\frac{\varphi_{t}}{2}\right)}{\sqrt{\left(\omega_{t}^{2}-\omega^{2}\right)^{2}+\left(2 \omega_{t} \eta_{t} \omega\right)^{2}}} \pm \frac{\sin (\omega \tau / 2) \cos \left(\omega t+\frac{\varphi_{r}}{2}\right)}{\sqrt{\left(\omega_{r}^{2}-\omega^{2}\right)^{2}+\left(2 \omega_{r} \eta_{r} \omega\right)^{2}}}\right\}
\end{aligned}
$$

where $P(t), a, c, m, \tau, \varphi_{t}$ and $\varphi_{r}, \omega, \omega_{t}$ and $\omega_{r}$, and $\eta_{t}$ and $\eta_{r}$ are the sound pressure, the diaphragm radius, the radius of central support, the mass of the diaphragm, the time delay factor, the translationaland rocking-mode phases, the operating frequency, the translational- and rocking-mode resonant frequencies, and the translational- and rocking-mode damping ratios, respectively. The theoretical model for elaborating the dynamic response is likewise verified by experimental measurements depicted in Fig. 4. Excellence match between calculation and measurement results indicates the accuracy of presented model and about 30\% net diaphragm displacement improvement. Additionally, the performances of a biomimetic microphone can be also strictly compared using two mechanical indicators, the mechanical interaural intensity difference per area (mIIDpA) and the mechanical interaural phase difference (mIPD) [1], which can be obtained as follows:

$$
\operatorname{mIIDpA}=\left[20 \log _{10} \frac{\left|Z_{\text {ipsi }}\right|}{\left|Z_{\text {contra }}\right|}\right] / \pi\left(a^{2}-c^{2}\right), \quad \operatorname{mIPD}=\angle \frac{Z_{\text {contra }}}{Z_{\text {ipsi }}}
$$


Table 1: Dimension parameters of the hybrid and CP-S design

\begin{tabular}{c|c}
\hline \hline \multicolumn{2}{c}{ Hybrid design } \\
\hline Radius of diaphragm $(a)$ & $1500 \mu \mathrm{m}$ \\
\hline Thickness of diaphragm $(T)$ & $5 \mu \mathrm{m}$ \\
\hline Length of edge-clamped supporting beam $(L)$ & $250 \mu \mathrm{m}$ \\
\hline Length of central beam $(L)$ & $250 \mu \mathrm{m}$ \\
\hline Width of beam $(W)$ & $15 \mu \mathrm{m}$ \\
\hline Spacing between beams & $15 \mu \mathrm{m}$ \\
\hline \hline CP-S design \\
\hline Radius of diaphragm $(a)$ & $1500 \mu \mathrm{m}$ \\
\hline Thickness of diaphragm $(T)$ & $5 \mu \mathrm{m}$ \\
\hline Radius of CP region $(c)$ & $225 \mu \mathrm{m}$ \\
\hline Radius of ring & $270 \mu \mathrm{m}$ \\
\hline Width of ring & $30 \mu \mathrm{m}$ \\
\hline Length of beam $(L)$ & $30 \mu \mathrm{m}$ \\
\hline Width of beam $(W)$ & $30 \mu \mathrm{m}$ \\
\hline \hline
\end{tabular}
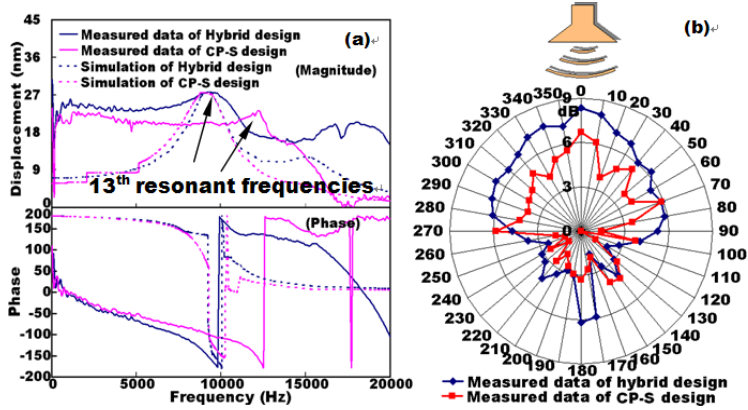

Figure 2: Comparison of (a) the frequency spectrum and (b) the logarithmic polar patterns of net diaphragm displacements between the hybrid and CP-S design.

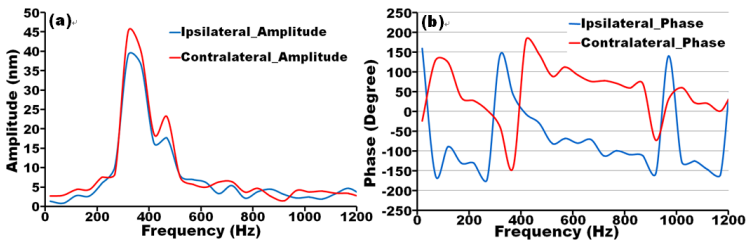

Figure 3: Acoustic responses of hybrid design in frequency domain in terms of (a) amplitudes and (b) phases of ipsilateral and contralateral of diaphragm, respectively.

For instance, the ipsi- and contralateral membranes move in opposite directions with equal amplitudes must have the mIIDpA and $\mathrm{mIPD}$ with the values of near $0 \mathrm{~dB} / \mathrm{mm}^{2}$ and $180^{\circ}$, respectively. Table 2 shows the comparisons in terms of nature frequencies and mechanical performances between the parasitoid fly [2], the conventional designs $[1,3,4]$ and the new structure. The presented hybrid design with the state-of-the-art characteristics of mIIDpA $2.7 \mathrm{~dB} / \mathrm{mm}^{2}$ and $\mathrm{mIPD} 155^{\circ}$ has shown a highly potential application for the sound source localization with full space sensitivity.

In summary, we develop a hybrid biomimetic microphone with a central floating pivot support. Better acoustic sensitivity and directivity can be realized theoretically and experimentally. The hybrid structure can not only inherit the advantages from conventional CP-S design, but also promote sound source localization sensitivity for MEMS microphones with a potential application for hearing aid devices [1].

\section{REFERENCES}

[1] H. J. Liu, M. Yu, and X. M. Zhang, "Biomimetic optical directional microphone with structurally coupled diaphragms," Applied Physics Letters, 93, 243902-1 (2008).

[2] H. Liu, L. Currano, D. Gee, B. Yang, and M. Yu, "Fly-Ear
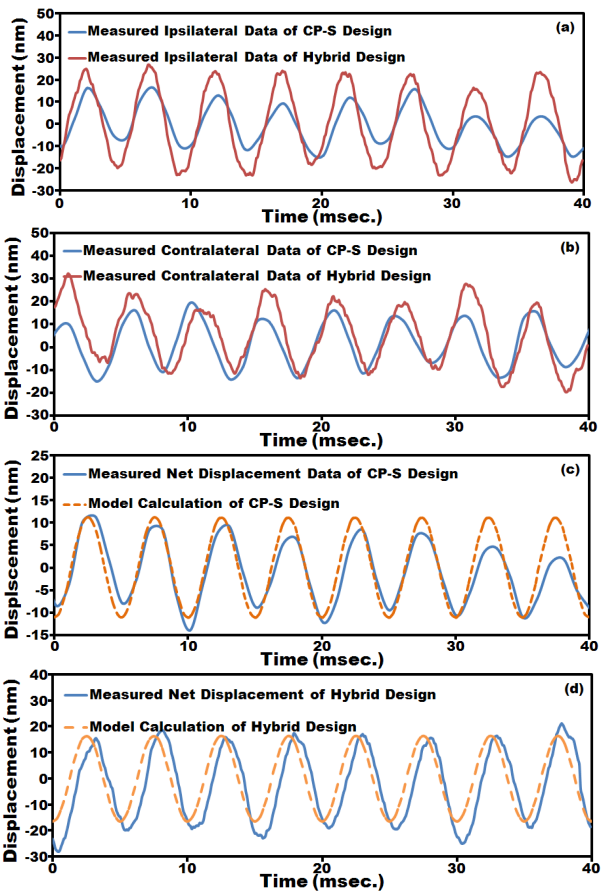

Figure 4: Measured (a) ipsilateral and (b) contralateral results of hybrid and $C P-S$ design, respectively. Comparison of model and net displacement are of (c) CP-S and (d) hybrid design, respectively.

Table 2: Comparisons in terms of nature frequencies and mechanical performances.

\begin{tabular}{|c|c|c|c|c|c|}
\hline Comparison & $\begin{array}{c}\text { First } \\
\text { translational } \\
\text { mode } \\
\text { frequency }\end{array}$ & $\begin{array}{l}\text { First } \\
\text { rocking } \\
\text { mode } \\
\text { frequency }\end{array}$ & $\begin{array}{c}\mathrm{mIIDpA} \\
\left(\mathrm{dB} / \mathrm{mm}^{2}\right)\end{array}$ & $\begin{array}{l}\text { mIPD } \\
(\mathrm{deg})\end{array}$ & $\begin{array}{c}\text { Full } \\
\text { space } \\
\text { sensing }\end{array}$ \\
\hline $\begin{array}{c}\text { Parasitoid } \\
\text { fly [2] }\end{array}$ & $31 \mathrm{KHz}$ & $7.1 \mathrm{KHz}$ & 10.0 & 80 & Yes \\
\hline Ref. [1] & $2.0 \mathrm{KHz}$ & $1.2 \mathrm{KHz}$ & 0.5 & 95 & No \\
\hline Ref. [3] & $0.7 \mathrm{KHz}^{*}$ & $0.2 \mathrm{KHz}^{*}$ & 2.7 & 135 & Yes \\
\hline Ref. [4] & $2.0 \mathrm{KHz}$ & $\mathrm{X}$ & 10.5 & $\mathrm{X}$ & No \\
\hline New design & $1.8 \mathrm{KHz}^{*}$ & $0.7 \mathrm{KHz}^{*}$ & 2.7 & 155 & Yes \\
\hline
\end{tabular}

$*:$ theoretical derivation; $\mathrm{X}:$ not available

Inspired Acoustic Sensors for Gunshot Localization," Proc. of SPIE, 7321, 73210A-1 (2009).

[3] N. Ono, A.Satio, and S. Ando, "Design and experiments of bio-mimicry sound source localization sensor with gimbal-supported circular diaphragm," Conference on Solid State Sensors, Actuators and Microsystems, Boston (2003), pp.935-938.

[4] W. Cui, B. Bicen, N. Hall, S. A. Jones, F. L. Degertekin, and R. N. Miles, "Optical Sensing Inadirectional Memsmicrophone Inspired by the Ears of the Parasitoid Fly, Ormia Ochracea," Proc. IEEE MEMS, 614 (2006).

[5] Y. C. Chen, C. C. Chen, Wen Hao Ching and Y. T. Cheng, "Design and Fabrication of High Performance Biomimetic Microphone Using Oxalis-Like Sensing Diaphragm for Sound Localization," APCOT Digest, 1B2-1(2008).

[6] CoventorWare, http://www.coventor.com/, version 2008.

\section{CONTACT}

${ }^{*}$ C. C. Chen, Tel: $+886-3-5712121$

\#54223; gettgod.ee92g@nctu.edu.tw 